\section{Metrología de la incertidumbre: un estudio de las estadísticas vitales en Chile y Brasil}

\author{
The metrology of uncertainty: a study of vital \\ statistics from Chile and Brazil
}

IEscuela de Salud Pública,
Universidad de Chile,
Santiago, Chile.
Correspondencia
Y. Carvajal
Escuela de Salud Pública,
Universidad de Chile.
Independencia 939, Santiago,
Región Metropolitana
83800oo, Chile.
ycarvajal@med.uchile.cl

Abstract

This paper addresses the issue of uncertainty in the measurements used in public health analysis and decision-making. The Shannon-Wiener entropy measure was adapted to express the uncertainty contained in counting causes of death in official vital statistics from Chile. Based on the findings, the authors conclude that metrological requirements in public health are as important as the measurements themselves. The study also considers and argues for the existence of uncertainty associated with the statistics' performative properties, both by the way the data are structured as a sort of syntax of reality and by exclusion of what remains beyond the quantitative modeling used in each case. Following the legacy of pragmatic thinking and using conceptual tools from the sociology of translation, the authors emphasize that by taking uncertainty into account, public health can contribute to a discussion on the relationship between technology, democracy, and formation of a participatory public.

Uncertainty; Vital Statistics; Cause of Death
Yuri Carvajal 1

Miguel Kottow ${ }^{1}$

\section{Introducción}

Este trabajo surge del propósito de probar la posibilidad e implicancias de la aplicación de una regla metrológica básica - la expresión de incertidumbre - al conteo de causas de muertes en el registro de defunciones.

Proponer a la salud pública cumplir un estándar metrológico, ampliamente usado en otras disciplinas que basan en la medición sus esfuerzos de investigación, es un experimento que propone:

- Una mejor especificación de lo "fuzzy" 1 que son muchas de las cifras usadas para tomar decisiones políticas de salud, y la factibilidad de pequeñas modificaciones que reduzcan esa incertidumbre presente en los datos.

- Comprender los aspectos performativos de los sistemas de medición y el rol de la metrología en la estabilización de hechos, que no poseyendo una positividad por sí mismos, surgen de estrategias constructivas.

- Introducir en salud pública comprensiones mundanas diferentes a la oposición de lo natural y social como opuestos y excluyentes.

- Discutir las dificultades democráticas del accionar a través del gobierno con números $2 \mathrm{o}$ aritméticas políticas y la relevancia de la construcción de un público.

El trabajo toma por objeto el conteo de causas de muerte en las estadísticas vitales, pues son cifras ampliamente conocidas, de acceso públi- 
co, usadas de forma directa o transformadas en tasas, años de vida saludable, años de vida potenciales perdidos $\mathrm{u}$ otra forma, para decisiones relevantes. Pese a que se elaboran con un énfasis muy especial en la reducción de su incertidumbre, no existe consenso en el reconocimiento de esa labor como de investigación o siquiera metrológica, en salud pública.

Mediciones de una especie de "universo" o realización única de un evento aleatorio irrepetible, de modo que la discusión sobre incertidumbre no está centrada en la inferencia y la especificación probabilística de error aleatorio y sistemático.

El resultado de estrategias constructivas acordadas en una comunidad de investigadores formada por humanos y no humanos (objetos, equipos, clasificaciones, regulaciones) y con la pretensión de lo que Dewey llamaba "asertibilidad garantizada" 3 o "afirmabilidad justificada" 4 . Las causas de muerte relevantes son la estabilización de controversias desplegadas por distintos actantes, objetos por lo tanto de un debate cuyas trazas no están a la vista, como en una "caja negra". Por tanto, susceptibles de ser reabiertas, reconociendo en ellas tensiones constituyentes y condiciones de posibilidad de otras alternativas.

\section{Marco teórico}

\section{Metrología e incertidumbre}

La metrología, ciencia de las mediciones y sus aplicaciones 5 , como disciplina individualizada y reconocida bajo esa denominación, data de los años sesenta. Sin embargo, la ocupación en la precisión y reproducibilidad de las mediciones es parte constitutiva del desarrollo de la ciencia.

La metrología se concentra en varios problemas vinculados con las mediciones, tales como magnitudes, sistemas de unidades, procedimientos y resultados, exactitudes, trazabilidad, validación, correcciones.

La especificación de incertidumbre proporciona valor, utilidad y comparabilidad a las mediciones: "When reporting the result of a measurement of a physical quantity, it is obligatory that some quantitative indication of the quality of the result be given so that those who use it can assess its reliability. Without such an indication, measurement results cannot be compared, either among themselves or with reference values given in a specification or standard. It is therefore necessary that there be a readily implemented, easily understood, and generally accepted procedure for characterizing the quality of a result of a measu- rement, that is, for evaluating and expressing its uncertainty" ${ }^{6}$.

La definición de incertidumbre no apela a una magnitud especificada como "valor verdadero [....sino a] un intervalo de valores que pueda ser razonablemente asociado al resultado" 5 . Esto implica un ejercicio constructivo en el cual no se propone una cifra preexistente e independiente de las mediciones, sino una aproximación empírica, a partir de los datos disponibles y la elaboración a partir de los mismos de una cifra para el contraste, que culmina en la especificación de incertidumbre: "En el enfoque en torno al concepto de error, el valor verdadero de la magnitud se considera único y en la práctica, imposible de conocer en la descripción de la medición" 5.

La incertidumbre de una medida es definida en las normas metrológicas como un parámetro no negativo que caracteriza la dispersión de los valores atribuidos a un mensurando, a partir de la información que se utiliza.

La evaluación de la incertidumbre considera tres aspectos básicos: (1) una definición clara del mensurando; (2) una especificación comprensiva del procedimiento de medida y; (3) un análisis comprensivo del impacto sobre los resultados de las mediciones.

En cuanto al abordaje de la especificación de la incertidumbre, se aceptan cuatro enfoques: (1) evaluación estándar o mediante modelación - construyendo una formulación de la influencia de cada factor a partir de la cual calcular una incertidumbre total como combinación de las incertidumbres estandarizadas; (2) validación del método - realizando mediciones repetidas, modificando los factores que influyen para estimar el sesgo; (3) validación interlaboratorios - comparando laboratorios para estimar repetibilidad, reproducibilidad y veracidad, medida respecto de un valor de referencia y; (4) test de competencia o Evaluación Externa de Calidad (EQA) - usando la variabilidad entre laboratorios incluidos en el programa como estimador de la desviación estándar de la reproducibilidad.

En el primer enfoque - conocido como evaluación de incertidumbre tipo A - es crucial la identificación y modelación de las fuentes de incertidumbre. Las evaluaciones tipo B, que constituyen las alternativas restantes, usan estimaciones obtenidas por la experiencia o información externa. El método conduce a expresar la incertidumbre a partir de una media y una varianza que tienen una cierta distribución probabilística.

En este caso tal modelación es compleja dado que los conteos y clasificaciones de defunciones se efectúan sólo una vez por cada evento, obteniendo una realización de la medida en cuestión, irrepetible. 
Entropía como aproximación a la incertidumbre

\section{- Información e incertidumbre}

Otra aproximación a la incertidumbre puede ser realizada a partir de la teoría de la información, haciendo uso de las medidas de entropía. Para Kullback 7 la teoría de la información es una rama de las teorías matemáticas de la probabilidad y estadística, constituida en torno a los aportes de Fisher, Shannon y Wiener. Su raíz matemática está en el concepto de entropía y considera que los trabajos de Shannon y Wienner conciernen a la teoría estadística de la información y que el uso de medidas de entropía, en teoría de transmisión de señales, es secundario respecto al uso como medida de información.

A partir de esos considerandos, la expresión más usada para aproximarse a la incertidumbre es la medida de entropía de Shannon, propuesta en 1948 como una medida de la entropía de un sistema, dado sus estados probables 8 :

$$
H=-\sum_{i=1}^{\mathrm{n}} p i \cdot \ln p i
$$

En un estado de máxima entropía, la información del sistema es máxima, con todos los estados posibles en condiciones de equiprobabilidad, por lo que $\mathrm{H}$ alcanza su valor pico. A la medida de entropía de Shannon se la considera una medida Hartley, que surge a partir de la medida propuesta por Hartley en 1928, que es 8:

$$
\begin{aligned}
& H\left(r_{E}\right)=\log _{2} \sum_{x \in X}^{\mathrm{n}} r_{E}(\mathrm{x}) \\
& \text { Con, } r_{e}(x)=\left\{\begin{array}{llll}
1 & \text { si } & x & \in E, \\
0 & \text { si } & x & \notin E
\end{array}\right.
\end{aligned}
$$

Esta medida tiene la propiedad de ser la única medida funcionalmente significativa posibilística de incertidumbre en bits 8 , pero adolece de la dificultad de no considerar las frecuencias de ocurrencia de cada resultado. Sus unidades pueden ser bits cuando la base de los logaritmos es dos. Si usamos base e son nats y bans cuando la base es 10. Un nat corresponde a 1.44 bits o 0.434 bans.

La medida de entropía de Shannon resuelve ese problema, ponderando el logaritmo de la probabilidad por su probabilidad.

\section{- Entropía y biodiversidad}

La ecología ha realizado una traducción de la medida de Shannon para especificar biodiversidad en ecosistemas. Asimilando las distintas especies del ecosistema a estados probables y sus frecuencias relativas, a sus probabilidades asociadas, se utiliza para compararlos respecto de la riqueza de especies y su distribución 9,10. Un valor máximo de la medida implica un ecosistema con las distintas especies igualmente representadas.

La medida de entropía anuda dos conceptos diferentes: riqueza de especies (S) y estructura (equiparidad: "evenness") de los ecosistemas (E). Un crecimiento en este índice puede deberse a un crecimiento de la riqueza de especies y/o de la estructura. Esta desventaja, empero, puede transformarse en ventaja si consideramos que una medida de estructura es:

$$
E=e^{H / s}
$$

Si tomamos logaritmos naturales:

$$
\ln E=H-\ln S
$$

Reacomodando: $\mathrm{H}=\ln S+\ln E$

\section{- Entropía y causas de muerte}

Volviendo a traducir esta medida a las causas de defunción, podemos especificar la entropía o información disponible en un conjunto de datos, usando las causas como estados probables y sus frecuencias, como probabilidades asociadas. La medida de entropía de Shannon podría ser interpretada como la cantidad de información adicional que requiero para conocer la causa de defunción de un caso seleccionado al azar dentro de la base de datos. Aplicada a la base de defunciones, una entropía x medida en bits o nats, significa que extraída al azar una defunción de la base correspondiente a un año, requiero $x$ bits o nats de información para poder saber de qué causa ha fallecido ese individuo. De esa manera, la entropía expresa la riqueza de información contenida en un determinado momento cronológico y puede ser usada como una aproximación indirecta a la incertidumbre (Tabla 1).

Aplicada a los datos de Chile, que oscilan en torno a 90.000 registros y 2.000 diagnósticos al año, la cifra máxima en condiciones de equiprobabilidad es alrededor de 12 bits. No obstante, ese máximo es moderadamente obvio y concordante: una equiprobabilidad de diagnósticos no implica información adecuada. En el lado de la cota inferior, con un sólo diagnóstico comprendiendo los 90.000 casos, $\mathrm{H}$ resulta ser 0 . Ese valor no es un óptimo de reducción de incertidumbre, pues todos los diagnósticos serían codificados en una sola causa de muerte. Eso también representa una extremada ignorancia, nula información y una gran incertidumbre.

La respuesta a la pregunta acerca de cuáles son las magnitudes de $\mathrm{H}$ deseables, sólo puede surgir a partir de mediciones parciales de entro- 
Tabla 1

Tres usos de la medida de Shanon.

\begin{tabular}{lccc}
\hline Unidad mínima & $\begin{array}{c}\text { Información } \\
\text { Señal }\end{array}$ & $\begin{array}{c}\text { Ecología } \\
\text { Individuos }\end{array}$ & $\begin{array}{c}\text { Datos DEIS } \\
\text { Defunciones }\end{array}$ \\
\hline I & Mensaje & Especies \\
$p_{i}$ & $\begin{array}{c}\text { Probabilidad de transmisión } \\
\text { del mensaje }\end{array}$ & $\begin{array}{c}\text { Frecuencia de individuos } \\
\text { en la especie } i\end{array}$ & $\begin{array}{c}\text { Causas de muerte } \\
\text { Frecuencia de defunciones } \\
\text { en la causa i }\end{array}$ \\
\hline
\end{tabular}

DEIS: Departamento de Estadísticas e Información en Salud.

pía en subconjuntos de datos, intentando identificar variantes que nos muestren como se mueve la entropía entre dos condiciones de las cuales podamos suponer razonadamente diferencias de calidad de los datos, mayor información y menor incertidumbre.

\section{Sistemas de codificación diagnóstica}

\section{- Clasificación internacional} de enfermedades

El método en uso para especificar la causa originaria de defunción es la Clasificación Estadística Internacional de Enfermedades y Problemas Relacionados con la Salud (dado que en Chile se usa desde 1997 la décima revisión para la codificación, se entenderá por tal la expresión CIE) tiene una historia que expresa el predominio taxonómico en el trabajo estadístico ${ }^{11}$. La organización taxonómica del conocimiento ha sido destacada, tanto en el origen de la medicina clínica 12 , como en la organización más global de los saberes, siendo su despliegue un momento destacable del paso de la época clásica a la moderna 13 . El rol performativo de este verdadero esquema clasificatorio se refleja en una estructuración que pone en un mismo plano causas por localización (sistemas involucrados), agentes (infecciosos) y lesión anátomo-patológica (cáncer). Destaca una especificación extrema de las condiciones de los accidentes de tránsito. Además de un isomorfismo de los trastornos mentales y del comportamiento (F00-F99) con las clasificaciones de la American Psychiatric Association, organizadas en el Diagnostic and Statistical Manual of Mental Disorders (DSM).

Mirko Grmek cuestionó la CIE-9 con criterios que siguen siendo válidos respecto a la versión actual: "Les publications médicales des deux derniers siècles contiennent la description d'environ 20.000 maladies, syndromes, formes de traumatismes et infirmités dignes de porter una étiquette particulière. Toutefois, un grand hôpital avec tous les services spécialisés n'enregistre généralement pas plus de 800 diagnostics différents et un médecin généraliste, même très avisé, se sert au maximum de 300 diagnostics" 14 (p. 17).

\section{- Nomenclaturas}

A partir del siglo XX, el vocabulario médico controlado empieza a aparecer como una alternativa al uso de taxonomías. Basado en la posibilidades de trabajar con reglas sintácticas complejas, tras las cuales subyace una ontología conceptual, disponen en el caso de Systematized Nomenclature of Medicine Clinical Terms (SNOMED CT) de más de 311.000 conceptos y de 1.360 .000 relaciones entre conceptos, constituyendo entramados conceptuales que prometen trazar configuraciones de la morbi-mortalidad radicalmente diferentes a las que hoy usamos.

\section{Material y método}

El trabajo se realizó en tres etapas: (1) estudio del proceso material de traducción de los datos de las defunciones; (2) exploración de las bases de datos de defunciones de Chile los años 1997 al 2008 y; (3) medición de entropía en la serie de datos, ajuste por tamaño mediante muestreo y cálculo de los componentes totales $(\mathrm{H})$, riqueza $(\ln S)$ e igualdad $(\ln E)$.

\section{Exploración de las bases de datos de defunciones 1997-2008}

Escogidas las bases de datos de defunciones de carácter semi-oficial entre el período 1997 al 2008, fueron descargadas desde el sitio http:// deis.minsal.cl. El período elegido corresponde a la serie codificada en la décima versión de la CIE. Las bases en formato dbf, fueron llevadas a formato dta (Stata Corp., College Station, USA) mediante Stat/Transfer v 7.0 (Circle Systems, Seattle, USA). La exploración de las bases se realizó cons- 
truyendo un "template" o plantilla de Stata v. 10 en modo "batch".

La exploración consideró: búsqueda de duplicados; etiquetado de variables y valores (label, values); recodificación de valores perdidos a [.]; búsqueda de inconsistencias en valores; pesquisa de valores sin código.

A partir de la plantilla, se construyeron códigos de exploración (archivos do en Stata) para cada uno de los años de la serie de tiempo, ya que los campos no mantienen los nombres a lo largo de la secuencia, trabajando los datos sin modificar la versión original de la base. También incorpora trazabilidad al procedimiento de exploración de los datos y trabajo de comunidad, pues el intercambio de los códigos de programación puede ser realizado a distancia vía internet, ya que los archivos son pequeños y fáciles de comprender. La plantilla incluye un archivo de salida (log en Stata) que escribe un archivo de texto con todos los hallazgos, procesable desde una amplia gama de procesadores de texto.

\section{Medición de entropía en los datos}

\section{- Serie de tiempo y parcialidades}

Se construyó un programa (archivo ado en Stata) que calcula la medida de entropía de cualquier variable.

Para obtener una interpretación de las mediciones de entropía, de acuerdo a lo ya señalado, respecto de la imposibilidad de obtener de la medida una correlación directa con incertidumbre, decidimos comparar distintas dimensiones de los datos, buscando un patrón de las mediciones, consistentes con patrones inferibles de incertidumbre. Las mediciones se hicieron según: años de 1997 a 2008; edad del difunto; región y servicio de salud de residencia; lugar de ocurrencia de la defunción (hospital, clínica o casa habitación); calidad de quien certifica (si es el médico tratante, legista-patólogo, otro, testigo o ignorado); comunas de residencia; nivel socioeconómico: medido en actividad y ocupación, en este caso, entre profesionales y trabajadores no cualificados; lugar de residencia (rural o urbano); datos de Brasil en cuanto a lugar de ocurrencia y condiciones como: color de piel en São Paulo.

\section{- Ajuste por tamaño}

Dado que los casos son diferentes en estas parcialidades, usando un criterio arbitrario de un 15\% como punto de corte, se comparó la entropía entre la parcialidad de menor tamaño (n), frente a la media de 1.000 muestras repetidas de tamaño $n$, tomadas en la parcialidad con mayor número de casos. A cada una de las 1.000 muestras se les midió la entropía y la riqueza. Se probó la estabilidad de ambos valores en los muestreos, graficando ambos valores. Este método se usó en la comparación entre regiones, servicios de salud, áreas rural y urbana, profesionales y trabajadores no cualificados, color de piel (São Paulo).

\section{- Separación entre riqueza $(\ln S)$ y estructura ( $\ln E)$}

Calculada en los muestreos la entropía media y la riqueza de diagnósticos, es posible calcular el aporte dado por la estructura como la sustracción del aporte de la riqueza a la magnitud de la entropía total.

\section{Resultados}

\section{Incertidumbre al clasificar/codificar}

Revisado el proceso de codificación, identificamos tres zonas de incertidumbre en la construcción de causas de defunción: (1) la actividad de diagnóstico médico y su registro manual en el certificado de defunción; (2) la actividad de registro del Servicio de Registro Civil (SRC) en sus oficinas nacionales y; (3) la actividad de codificación en el Departamento de Estadísticas e Información en Salud (DEIS).

Incertidumbres en los datos desde un punto de vista descriptivo

Las bases tienen 51 campos y aproximadamente 90.000 registros por año. El primer hallazgo a destacar es que los campos no tienen el mismo orden en todo el período, ni la misma denominación cada año. Algunos desaparecen o se transforman en otros. La mayoría de los problemas están vinculados con los datos construidos por el SRC. Hay casos sin fecha de nacimiento o día de defunción todos los años de la serie.

La codificación de actividad y ocupación se refiere al momento de la defunción, por lo que resulta de poca utilidad, ya que en promedio el $83 \%$ de los casos son codificados como inactivos.

En la exploración se pesquisaron otras anomalías menores: incongruencias entre la cifra de hijos totales, comparada con la suma de hijos fallecidos e hijos vivos; existe preferencia de dígito en la fecha de nacimiento y en fecha de muerte; la variable edad de la madre toma un valor 0 para representar no corresponde, al igual que en hijos vivos y fallecidos; la variable fecha de parto o aborto anterior, que corresponde a la fecha de 
ocurrencia de un parto o aborto anterior al caso registrado, en muchas ocasiones registra la fecha de nacimiento del caso; en atención médica se encontraron valores (5) que no poseen codificación y; en ocupación se ocupa indistintamente $\mathrm{x}$ oX.

Un problema que puede ser resuelto muy sencillamente, generando códigos de programación que modelen la base antes de su publicación con el cálculo de las edades. La base utiliza dos codificaciones diferentes, lo cual es complejo de manejar. No usa fechas como tales, de modo que la edad en algunos casos cada año difiere más de un año respecto de la calculada entre fecha de nacimiento y defunción.

La base de datos incluye los códigos alfa numéricos de CIE, los cuales no son fácilmente convertibles a la glosa correspondiente o nombre de la enfermedad, por un usuario que no tenga el soporte para vincular ambos datos. Al hacerlo, en algunos pocos casos los códigos no tienen glosa CIE.

\section{Cuantificando incertidumbre: entropía}

\section{- Serie de tiempo}

La variación anual del número de diagnósticos cada año es pequeña, pero se incorporan y desusan unos 400 diagnósticos. Eso, sobre un total de 12.800 aproximadamente de códigos disponibles.

La Figura 1 muestra las variaciones de información de la serie de tiempos. El patrón es consistente con mejor calidad de la información junto con su disponibilidad en sitio web, a partir de una reorganización del DEIS, expresado en cinco parámetros de registros que se han modificado desde los años 2002-2003 en el siguiente sentido: incremento de las defunciones fetales; incremento de las muertes maternas; mejoría de la integridad de los datos de defunciones en menores de un año; reducción de causas mal definidas; e identificación de causas sometidas a vigilancia.

\section{- Hospitales y no hospitales}

A lo largo de toda la serie, las defunciones ocurridas dentro de los hospitales, de acuerdo a Figura 2 , tienen mayor información que las ocurridas en otros sitios.

Si separamos la entropía en sus dos componentes para esta diferencia, observamos que la información contenida en los datos hospitalarios es mayor en ambas dimensiones según Tabla 2.

Los datos hospitalarios corresponden a una población con un leve predominio masculino y de menor edad, respecto a los fallecidos en otros sitios (Tabla 3 ).

\section{- Profesionales y trabajadores no cualificados}

Entre profesionales y trabajadores no cualificados, ajustando el tamaño mediante muestreo, se observa que la información de las defunciones contiene mayor información en toda la serie, no

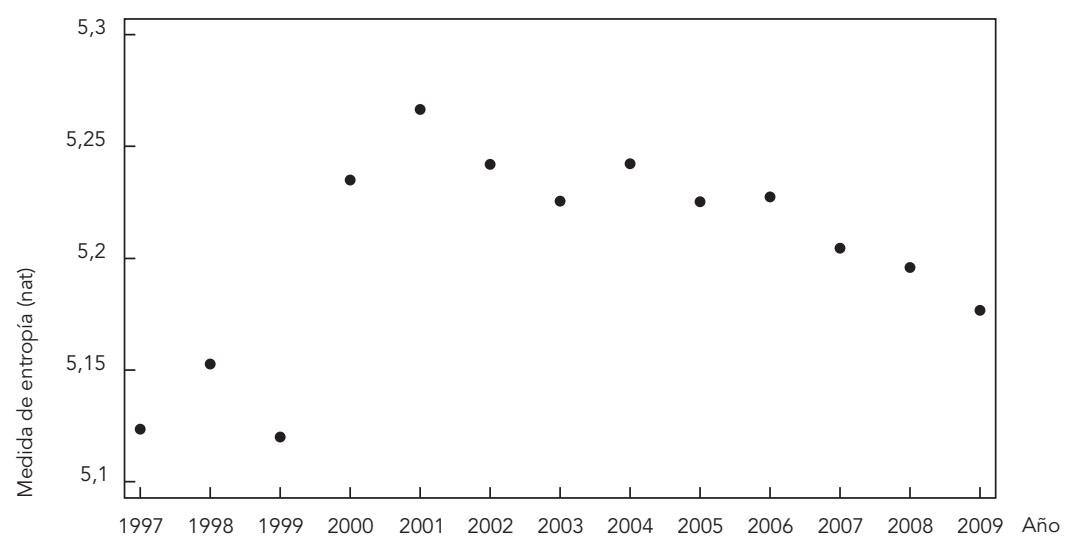




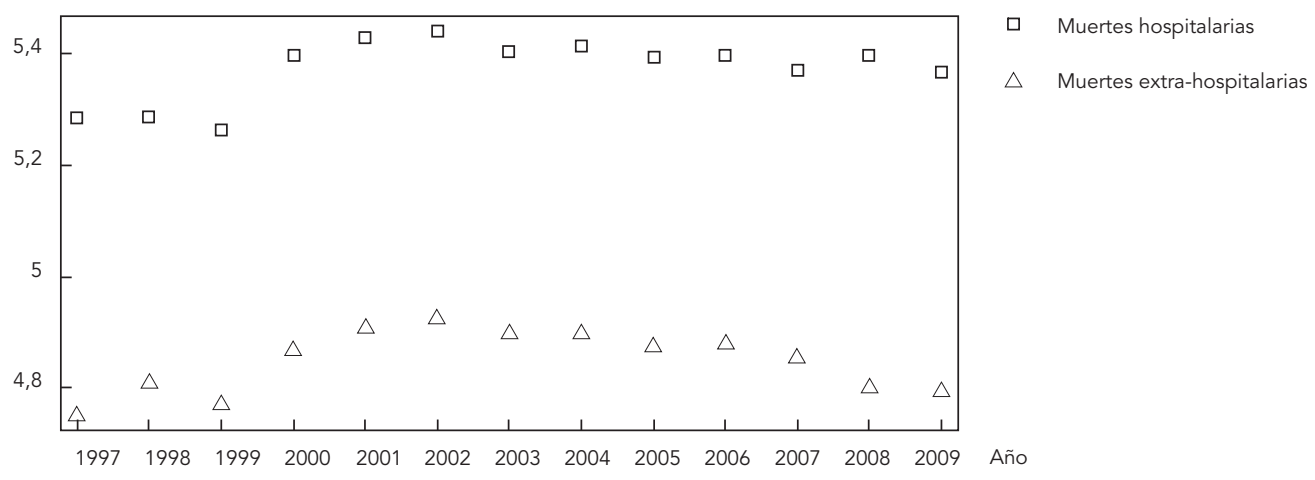

Tabla 2

Entropía, diversidad y estructura entre información contenida en defunciones ocurridas en y fuera de hospitales.

\begin{tabular}{lcc}
\hline Medida & Hospitales & No hospitales \\
\hline $\mathrm{H}$ & 5,3971413 & 4,8022803 \\
$\mathrm{~S}$ & 1.583 & 1.096 \\
$\mathrm{~N}$ & 42.606 & 47.562 \\
InE & $-1,9699358$ & $-2,1971422$ \\
InS & 7,3670771 & 6,9994225 \\
\hline
\end{tabular}

H: entropía total; InE: logaritmo natural de estructura ("evennness"); InS: logaritmo natural de riqueza de especie; $\mathrm{N}$ : número de casos o individuos; S: riqueza de especies (número de especies o diagnósticos en este caso). Fuente: Departamento de Estadísticas e Información en Salud (http://deis.minsal.cl).

Tabla 3

Comparación entre sexo y edad de las defunciones hospitalarias y extra hospitalarias.

\begin{tabular}{lcc}
\hline & Hospitales & No hospitales \\
\hline $\begin{array}{l}\text { Edad promedio } \\
\text { (años) }\end{array}$ & 68 & 72 \\
$\begin{array}{l}\text { Proporción de } \\
\text { hombres (\%) }\end{array}$ & 56 & 52 \\
\hline
\end{tabular}

Fuente: Departamento de Estadísticas e Información en Salud (http://deis.minsal.cl). sólo por la riqueza o variedad de diagnósticos, sino también por la estructura, según Figura 3.

\section{- Zonas rurales y urbanas}

Tras la realización de un muestreo en las defunciones de zonas urbanas, para equiparar la magnitud de zonas urbanas, tenemos una estimación consistente de entropía que distingue ambas áreas, siendo mayor la entropía de las defunciones de las zonas urbanas según se muestra en Tabla 4.

\section{Brasil}

Brasil provee a través de la página electrónica del Departamento de Informática do SUS (DATASUS. http://www2.datasus.gov.br/ DATASUS/index.php?area $=0205$, accedido el 02/May/2011) bases de datos de estadísticas vitales, administrables con el software TabWin (DATASUS. http://portal.saude.gov.br/portal/ se/datasus/area.cfm?id_area=732), desarrollado localmente y descargable en el mismo sitio.

Usando los datos del 2009, contrastamos la entropía de los datos en las defunciones hospitalarias y domiciliarias y por color de piel de la persona fallecida para el Estado de São Paulo (Figura 4). Este estado tiene casi el triple de defunciones por período que Chile. El patrón de las defunciones hospitalarias y domiciliarias en este estado es consistente con los hallazgos de Chile (Figura 5).

En ambas condiciones, cifras vinculadas con menor entropía se corresponden con color de piel oscura y con muertes domiciliarias. Los tamaños se corrigieron mediante muestreo. 


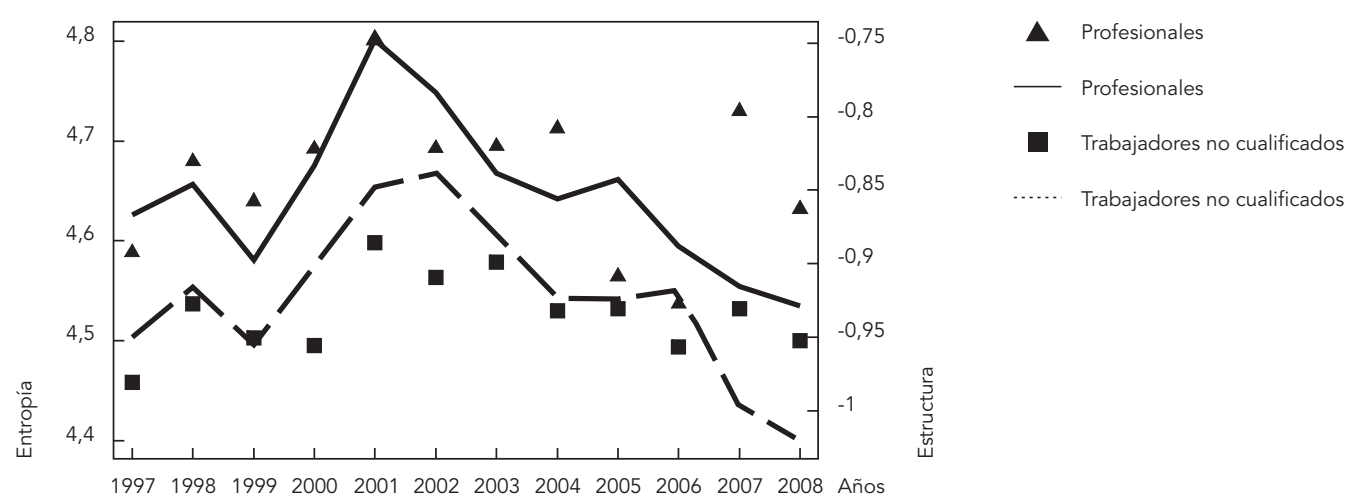

Tabla 4

Entropía de Shannon, ajustada por tamaño de muestra, en causas de defunción de zonas rurales y urbanas.

\begin{tabular}{lccc}
\hline Medida & Rurales & Urbanas & Media en muestras urbanas \\
\hline $\mathrm{H}$ & 4,9960857 & 5,2044881 & 5,1428467 \\
$\mathrm{~S}$ & 778 & 1.728 & 857 \\
$\mathrm{~N}$ & 11.378 & 78.790 & 11.378 \\
$\ln E$ & $-1,6606408$ & $-2,2502318$ & $-1,6108662$ \\
$\ln S$ & 6,6567265 & 7,4547199 & 6,753713 \\
\hline
\end{tabular}

H: entropía total; InE: logaritmo natural de estructura ("evennness"); InS: logaritmo natural de riqueza de especie; N: número de casos o individuos; S: riqueza de especies (número de especies o diagnósticos en este caso).

\section{Discusión}

Reducción de la incertidumbre en el proceso de traducción

\section{- Metrología en los tres momentos de la certificación}

El rol de los médicos a partir de la descripción y análisis del proceso de codificación y de un examen de la conformación de los datos en las bases, reconocemos que existen elementos no susceptibles de metrología, tales como las indeterminaciones del acto médico. No obstante, existe un amplio margen en la codificación y el procesamiento de los datos. El campo de trabajo contiene, por tanto, posibilidades y limitantes metrológicos. Si bien la posibilidad de introducir nuevas reglas en el proceso de registro es limita- do, dada la condición "civil" de tal actividad, un sistema de certificación online permitiría limitar y potenciar la capacidad del médico y a la vez incorporar trazabilidad a los datos. Incluso podría considerar adicionar antecedentes tenidos a la vista para hacer el diagnóstico, como ficha, exámenes, evolución clínica.

La capacidad de imponer reglas metrológicas al SRC desde el DEIS es limitada en el marco de las relaciones institucionales (dependencias jerárquicas distintas) y además el ingreso de diagnósticos y categorías ocupacionales es realizado por un funcionario cuyos conocimientos están orientados hacia otras especificidades. Una alternativa a un sistema online, es la vinculación con un archivo digital del documento emitido por el médico y la imposición obligatoria del registro de todos los campos especificados en el documento. 


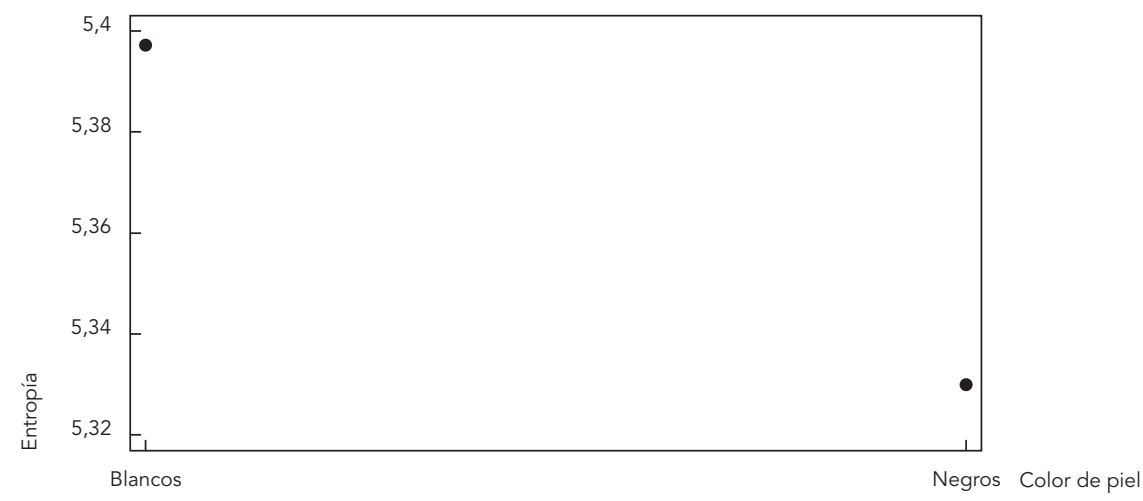

\section{Figura 5}

Entropía de causas de defunción, medida en domicilios y muestreada en hospitales. São Paulo, Brasil, 2009.

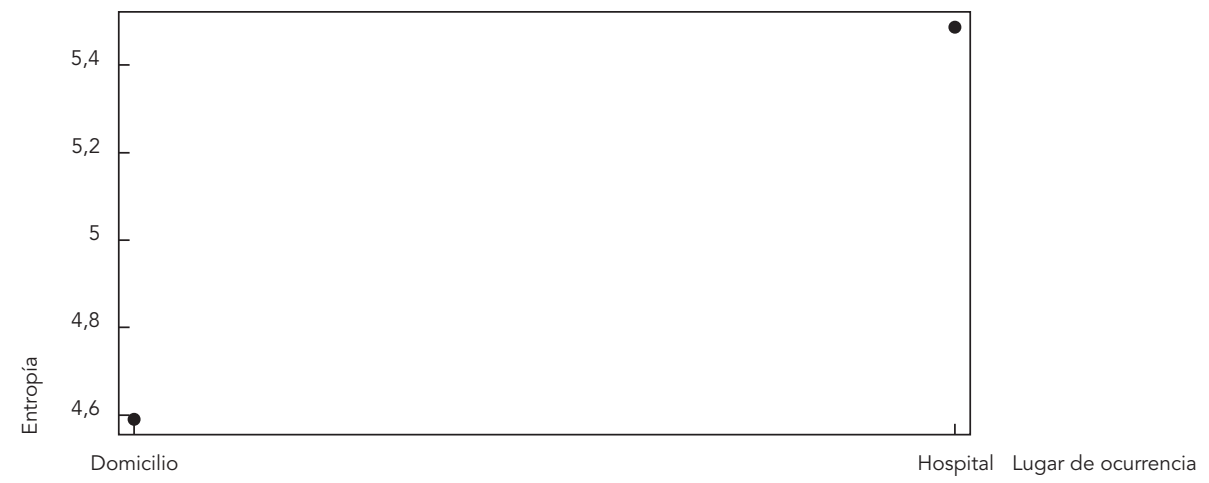

La zona de mayor posibilidad de introducir mejoras es el proceso mismo del DEIS, en donde cualquier automatización, ya sea de partes o de todo el proceso, permitiría validar campos, generar estándares, identificar inconsistencias, introducir medidas de calidad mediante trazabilidad de la autoría de las distintas transformaciones realizadas a los datos. Cualquier propuesta debe considerar una tríada compuesta de: manutención de todos los campos diagnósticos, codificación en varios campos - automatizada y por codificadora - y el uso simultáneo de CIE y SNOMED CT.
Otro elemento a relevar es la estandarización de la exploración de los datos. Existen buenos textos que justifican y orientan el valor y la utilidad de los procedimientos estandarizados y documentados 15,16. El reporte de hallazgos en un estudio, debiera informar los resultados de la exploración de los mismos como parte de la labor investigativa y la documentación al respecto debiera quedar disponible. Una aproximación sistemática al estudio de la organización de los datos y una especificación del proceso de revisión, puede ser organizada mediante sistemas de archivos con códigos de instrucciones, 
fácilmente replicables, los problemas detectados debieran ser comunicados. Una tarea modesta y laboriosa se puede transformar en una fuente de reflexión notable, acerca de lo que son los datos como objeto de estudio. Así como la prudencia ante las conclusiones y decisiones que a partir de ella se infieran o decidan.

\section{- Métrica de la incertidumbre}

Respecto de la componente cuantificación ensayada, la entropía de Shannon y la corrección realizada para las diferencias de tamaño, constituyen una primera aproximación a una comparación de la información al interior de un país. La interpretación que los descensos de la medida de entropía implican peores datos y, por tanto, pueden revestir utilidad para expresar incertidumbre. Esta forma de interpretar los hallazgos puede ordenarse del siguiente modo: la cifra máxima de información que registramos para las causas de defunción en una base de datos, en un lugar y momento, expresa una cantidad de incertidumbre relativa, situada bajo el mínimo o la mejor especificación esperable o teórica de causas, en ese momento, ya que todas las mediciones de $\mathrm{H}$, muestran un descenso consistente y sostenido en relación con algunos factores que, de acuerdo a la experiencia, agregan incertidumbre a los diagnósticos.

¿Porqué no aceptar que el máximo de cada año es simplemente la menor incertidumbre?

- Porque examinados el proceso de registro de las causas, hay marcadas áreas de incertidumbre algunas posibles al menos de ser trazadas (identificación de los médicos que certifican, el tipo de hospital) y otras, que aún sin identificar, aportan imprecisión a los datos.

- Porque examinados los datos es evidente que contienen incertidumbre que puede ser reducida mediante sencillas técnicas de administración de datos.

- Porque en los mismos campos diagnósticos, fuera de las causas mal definidas, hay algunos diagnósticos oscuros como hiperlipidemia no especificada, bloqueo de rama izquierda del haz de His, varicela sin complicaciones o infección viral no complicada. En el año 2008 encontramos un caso de carotinemia (E671), otro de pólipo anal (K620) y uno de hiperémesis gravídica leve (O210)

- Porque existen soluciones tecnológicas relativamente simples - tres de ellas absolutamente disponibles - que pueden hacer un uso pleno de la información actualmente disponible: SNOMEDCT, IRIS (sistemainteractivo de codificación de causas de muertes y de selección de causa básica) y certificado médico de defunción on line.
El rol de los hospitales en esta exploración, la información contenida en los diagnósticos aparece asociada fuertemente al tipo de establecimiento, que a la condición del médico respecto de su conocimiento del paciente como tratante. Esta dimensión puede vincularse con el patrón geográfico, los servicios, la ruralidad y quizás, también el patrón encontrado en la condición laboral. Si esto realmente es así, la precisión diagnóstica resulta asociada al dispositivo técnico del cual el médico es parte y no a su mera presencia o ausencia, como acompañante de la defunción.

En suma concluimos haber ensayado una medida relativa de incertidumbre y un método de ajuste por tamaño, cuya magnitud es específica y aplicable para este conjunto de datos, pero que debe ser probada en otras condiciones, para confirmar la propuesta de identificar variaciones de la incertidumbre de los datos a nivel regional, urbano-rural, de acuerdo a una categorización de la actividad, del sitio de defunción (hospital y no hospital). A la vez, hemos podido corregir las diferencias dadas por el tamaño de los registros, mediante muestreos aleatorios y separando dos componentes de la incertidumbre, riqueza y estructura.

\section{Performación de la medida}

El trabajo de reducción de incertidumbre señala la necesidad de ampliar el horizonte del análisis desde la cuestión de la exactitud, hacia la cuestión misma de la medida y de aquello que expresa la incertidumbre, no sólo como un problema de precisión de una medida, sino del acto de constitución de la dimensionalidad de lo que se mide. Medir es un acto estructurante, organizador; que especifica y construye dimensiones, las hace medibles. En esa operación, hay inclusión y exclusiones, hay organización y reglas, condiciones de posibilidad. Como dice Senra 17 (p. 16): "Las estadísticas no revelan, simplemente las realidades, tal como son; revelan realidades previamente construidas, idealizadas, construcciones que no son arbitrarias, personalistas, al contrario, son públicas y colectivas, obedeciendo a los dictámenes de las ciencias... son tanto del orden de la sintáctica como del orden de la semántica".

Por eso, la interrogación por la incertidumbre en salud pública y en particular aquella contenida en las cifras de defunciones tiene una relevancia que excede el afán de precisión. El reconocimiento de la incertidumbre permite avanzar a una reflexión de la práctica de investigación en el campo disciplinar.

Performación de la medida o de medida mensurante significa que una categoría, un código, una clasificación no existen como una idea 
pura, sino que forman parte de un dispositivo, en el cual se construyen los hechos. Se ensamblan y se articulan. Que en controversias científicas jamás hay lo dado, no sólo porque la teoría orienta aquello que distinguimos, sino porque merced a la proliferación de equipos, artefactos como teoría material organizada, como equipamiento, proponen una carga teórica. El hecho científico independiente y autónomo es el resultado de una deliberada construcción en pos de su autonomía. El apelativo dado por Latour de "factiche" para los hechos, explicita el carácter constructivo que poseen los mismos, surgidos de la articulación material de una práctica 18. Factiche, unión de hecho y fetiche, cuestiona la validez de distinciones entre aquello que es y aquello en lo que se cree. Negada la distinción que separa creencia de saber, experiencia de realidad, invalida el juicio de falsedad sobre una creencia no compartida. Factiche permite mostrar no sólo la dimensión pragmática del hecho, sino mostrar su carácter construido e híbrido. Latour 18 ha recurrido a la expresión de Serres, cuasi-objetos, aludiendo a los hechos como híbridos, como compuestos de una tríada de materialidad, significado y relaciones de fuerza. La exploración de la historia de los factiches, como forma de interrogarse por las diferentes transacciones y negociaciones que han acompañado su construcción, introduce una doble dimensión de existencia de los mismos. En un eje horizontal, el trabajo de purificación ("científico") que releva su autonomía y en la dimensión vertical, el trabajo de articulación o ensamblaje, que expresa su carácter de construido 18 .

Teniendo los factiches la doble constitución de lo construido y lo dado, cargando sobre sus espaldas su propia historia, de purificación e hibridación, los objetos estadísticos pueden ser comprendidos como tales. En el sentido horizontal como representaciones cada vez más verificables, trazables y replicables. Verticalmente como hibridaciones de signos, relaciones y objetos, elaborados como dispositivos o asociaciones.

\section{Natural versus social}

La expresión de incertidumbre en salud pública supone romper las barreras que separan lo natural y lo social, haciéndonos parte de un cuestionamiento no moderno a la modernidad. La metrología no es propia de uno u otro mundo, sino es la herramienta que permite identificar las redes que asocian distintos actores, la que insiste en la trazabilidad, en la posibilidad de comprender la inmutabilidad de los móviles, a través de su rastreabilidad en la larga cadena de traducciones. Sin embargo, también metrología es la que sienta las reglas para la construcción de estándares y pseudo estándares, que subyacen a los procesos de intercambio, de traducción, a las asociaciones entre actantes, posibilitando la diversidad, la creatividad y la innovación. No hay una metrología de lo natural versus una de lo social, sino reglas que permiten estabilizar hechos y componer un mundo colectivo común.

La dicotomía de actividad científica y de actividad social, escinde el trabajo de purificación de los cuasi-objetos de la historia de su constitución. Oculta las pugnas y acuerdos - las relaciones de fuerza - que han precedido su reconocimiento y aceptación por la comunidad de investigadores. La historia de las mediaciones es velada por la purificación, volviendo ardua la tarea de escrutar qué guardan en su interior los hechos científicos.

\section{Lo público de la salud pública}

Si lo social - como sugiere la sociología de la traducción 19 - no está dado, sino que debe ser compuesto, el énfasis público de la salud pública requiere la explicitación metrológica de sus cifras. Público como labor constructiva de un público, en el sentido en planteado por Dewey 20 (p. 311): "la necesidad esencial es la mejora de los métodos y condiciones de debate, de discusión y de persuasión. Este es el problema del público".

La construcción de un colectivo a través del debate de los problemas de salud y enfermedad - un público simultáneamente político y técnico - propone a la salud pública formas de democracias dialógicas, foros híbridos 21.

Metrología e incertidumbre en salud pública son a la vez herramientas de trabajo, objeto de estudio y requerimiento democrático de construcción de un público.

\section{Conclusiones}

El proceso de producción, médico y estadístico, de causas de defunción posee una imprecisión nosológica, niveles diferentes de pericia y conocimiento. Tras ese trasfondo, aparece una primera expresión de incertidumbre en las heterogeneidades en la información contenida por las bases de datos, según territorios, establecimientos y ocupaciones. Las cifras de salud pública no están exentas de incertidumbre, aunque su metrología esté pendiente.

Construir estadísticas no es reflejar una realidad preexistente, sino organizar, excluir, limitar, conducir. Las estadísticas tienen un importante rol performativo, que exige una labor de investigación, explicitación y expresión conceptual y numérica 17 . 
Reglas metrológicas pueden y deben aplicarse a las mediciones en salud pública. La revisión de la oposición social natural, aporta ventajas para una mejor comprensión del carácter de cuasiobjetos de las estadísticas y los aspectos controversiales que anidan en nuestro trabajo 18 .

Democracia, conocimiento, valores, igualdad, universalidad, técnica, individualismo, autonomía, pueden ser reexaminados a la luz de la sociología de las ciencias, y en particular de la identificación del experimento, permiten revisitar debates de metodología y diseño en salud pública, reflexionar sobre los orígenes de las aritméticas políticas de Petty y su conexión con los trabajos de Graunt 22, las filosofías morales de Smith o las ciencias morales de Condorcet. Sus aproximaciones a la incertidumbre 23 nos recuerdan que compartimos con la demografía, la economía, la estadística y la sociología 24 , el uso de la formalización matemática. Y que junto a los números, la incertidumbre expresa la aporía de unificar lo diverso, múltiple y plural.

Finalmente, todo lo anterior tiene valor, utilidad, sentido y se justifica, si acaso salud pública aspira a ser parte de un ejercicio de debate público y de un público, situándose como constructora de un mundo común.

\section{Resumen}

Este artículo examina la incertidumbre presente en una de las mediciones más usada para análisis y toma de decisiones en salud pública. Adapta la medida de entropía de Shannon-Wiener para expresar la in certidumbre contenida en el conteo de las causas de muertes en las estadísticas vitales oficiales de Chile. partir de los hallazgos, discute los requerimientos metrológicos en salud pública, tan importantes como las mediciones mismas. Considera y argumenta la exis tencia de una incertidumbre adicional, asociada con las propiedades performativas de las estadísticas. Tan to por la forma de estructurar los datos a la manera de una cierta sintaxis de lo real, como por las exclusiones de aquello que queda más allá de la modelación cuantitativa usada en cada caso. Mediante una aproximación a la herencia del pensamiento pragmático, y usando herramientas conceptuales de la sociología de la traducción, destaca que la incertidumbre puede contribuir en salud pública a un debate acerca de la vinculación entre técnica, democracia y la formación de un público.

Incertidumbre; Estadísticas Vitales; Causas de Muerte 


\section{Colaboradores}

Y. Carvajal desarrolló la idea cuantitativa central, organizó el material, la bibliografía y redactó la versión a ser publicada. M. Kottow propuso variantes analíticas y de interpretación de los datos, reorganizó la estructura, revisó la redacción y propuso modificaciones a la discusión, preparó la versión a ser publicada.

\section{Referencias}

1. Zadeh L. Fuzzy sets. Information and Control 1965; 8:338-53.

2. Desrosiéres A. Gouverner par les nombres: l'argument statistique II. Paris: Mines Paris Tech Les Presses; 2008.

3. Dewey J. El hombre y sus problemas. Buenos Aires: Editorial Paidós; 1954.

4. Rorty R. La filosofía y el espejo de la naturaleza. Madrid: Cátedra; 1989.

5. Joint Committee for Guide in Metrology. Vocabulario internacional de metrología (VIM): conceptos fundamentales y generales y términos asociados. http://www.bipm.org./en/publication/guides/ vim.htm (accedido el Ene/2010).

6. Joint Committee for Guide in Metrology. Evaluation of measurement data guide to the expression of uncertainty measure. http://www.bipm.org./utils/ common/documents/jcgm/JCGM_100_2008_E. pdf (accedido el Ene/2010).

7. Kullback S. Information theory and statistics. New York: Dover Publications; 1997.

8. Klir G. Uncertainty and information foundations of generalized information theory. New Jersey: Wiley-Interscience; 2006.

9. Moreno C. Métodos para medir la biodiversidad. Zaragoza: CYTED ORCYT-UNESCO/Sociedad Entomológica Aragonesa; 2001.

10. Jost L. Entropy and diversity. Oikos 2006; 113: 363-75.

11. Organización Panamericana de la Salud. Clasificación estadística internacional de enfermedades y problemas relacionados con la salud, décima revisión. Washington DC: Organización Panamericana de la Salud; 1995.

12. Foucault M. El nacimiento de la clínica. México DF: Editorial Siglo XXI; 1991.

\section{Agradecimientos}

A Sergio Alvarado, Escuela de Salud Pública, Universidad de Chile y Carlos Henríquez, Centro de Estudios Estadísticos, Universidad Valparaíso por su asesoría matemática y estadística.
13. Foucault M. Las palabras y las cosas: una arqueología de las ciencias humanas. México DF: Editorial Siglo XXI; 1993.

14. Grmek M. Declin et émergencie des maladies. Hist Ciênc Saúde-Manguinhos 1995; 2:9-32.

15. Baum C. An introduction to stata programming College Station: Stata Press; 2009.

16. Long S. The workow of data analysis using Stata. College Station: Stata Press; 2009.

17. Senra N. O saber e o poder das estatísticas. Rio de Janeiro: Instituto Brasileiro de Geografia e Estatística; 2005.

18. Latour B. Nous n'avons jamais été modernes. Paris: La Découverte/Poche; 1997.

19. Akrich M, Callon M, Bruno L. Sociologie de la traduction: textes fundateurs. Paris: Mines Paris Les Presses; 2006.

20. Dewey J. Le public et ses problèmes. Paris: Gallimard; 2005.

21. Callon M, Lascoumes P, Barthe Y. Agir dans un monde incertain: essai sur la démocratie technique. Paris: Editions du Seuil; 2001.

22. Bras HL. Naissance de la mortalité. France: Gallimard Le Seuil; 2000.

23. Rothschild E. Economic sentiments. Adam Smith, Condorcet and the enlightenment. Cambridge: Harvard University Press; 2001.

24. Latour B, Lepinay V. La economía: ciencia de los intereses apasionados. Buenos Aires: Manantial; 2009 .

Recibido el 21/Sep/2011

Aprobado el 07/Ago/2012 